\title{
IMF DIRECTION DERIVED FROM CYCLOID-LIKE ION DISTRIBUTIONS OBSERVED BY MARS EXPRESS
}

\author{
M. YAMAUCHI ${ }^{1, *}$, Y. FUTAANA ${ }^{1,7}$, A. FEDOROV ${ }^{2}$, E. DUBININ ${ }^{3}$, R. LUNDIN $^{1}$, \\ J.-A. SAUVAUD ${ }^{2}$, D. WINNINGHAM ${ }^{4}$, R. FRAHM ${ }^{4}$, S. BARABASH ${ }^{1}$,

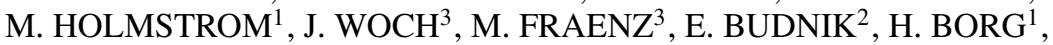 \\ J. R. SHARBER ${ }^{4}$, A. J. COATES ${ }^{5}$, Y. SOOBIAH ${ }^{5}$, H. KOSKINEN ${ }^{6,17}$, E. KALLIO ${ }^{6}$,
}

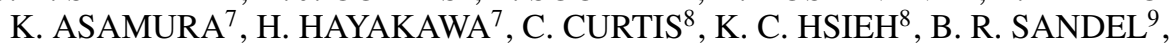
M. GRANDE ${ }^{10}$, A. GRIGORIEV ${ }^{1}$, P. WURZ $^{11}$, S. ORSINI $^{12}$, P. BRANDT $^{13}$, S. MCKENNA-LAWLER ${ }^{14}$, J. KOZYRA ${ }^{15}$ and J. LUHMANN ${ }^{16}$

${ }^{1}$ Swedish Institute of Space Physics, Box 812, SE-98128, Kiruna, Sweden

${ }^{2}$ Centre d'Etude Spatiale des Rayonnements, BP-4346, F-31028 Toulouse, France

${ }^{3}$ Max-Planck-Institut für Sonnensystemforschung, D-37191 Katlenburg-Lindau, Germany

${ }^{4}$ Southwest Research Institute, San Antonio, TX 7228-0510, USA

${ }^{5}$ Mullard Space Science Laboratory, University College London, Surrey RH5 6NT, UK

${ }^{6}$ Finnish Meteorological Institute, Box 503 FIN-00101 Helsinki, Finland

${ }^{7}$ Institute of Space and Astronautical Science, 3-1-1 Yoshinodai, Sagamichara, Japan

${ }^{8}$ Department of Physics, University of Arizona, Tucson, AZ 85721, USA

${ }^{9}$ Lunar and Planetary Lab, University of Arizona, Tucson, AZ 85721, USA

${ }^{10}$ Rutherford Appleton Laboratory, Chilton, Didcot, Oxfordshire OXI1 OQX, UK

${ }^{11}$ University of Bern, Physikalisches Institut, CH-3012 Bern, Switzerland

${ }^{12}$ Instituto di Fisica dello Spazio Interplanetari, I-00133 Rome, Italy

${ }^{13}$ Applied Physics Laboratory, Johns Hopkins University, Laurel, MD 20723-6099, USA

${ }^{14}$ Space Technology Ltd., National University of Ireland, Maynooth, Co. Kildare, Ireland

${ }^{15}$ Space Physics Research Lab., University of Michigan, Ann Arbor, MI 48109-2143, USA

${ }^{16}$ Space Science Lab., University of California in Berkeley, Berkeley, CA 94720-7450, USA

${ }^{17}$ University of Helsinki, Department of Physical Sciences, Box 64, FIN-00014, Helsinki, Finland

(*Author for correspondence: E-mail:m.yamauchi@irf.se)

(Received 6 April 2006; Accepted in final form 31 October 2006)

\begin{abstract}
Although the Mars Express (MEX) does not carry a magnetometer, it is in principle possible to derive the interplanetary magnetic field (IMF) orientation from the three dimensional velocity distribution of pick-up ions measured by the Ion Mass Analyser (IMA) on board MEX because pick-up ions' orbits, in velocity phase space, are expected to gyrate around the IMF when the IMF is relatively uniform on a scale larger than the proton gyroradius. Upstream of bow shock, MEX often observed cycloid distributions (two dimensional partial ring distributions in velocity phase space) of protons in a narrow channel of the IMA detector (only one azimuth for many polar angles). We show two such examples. Three different methods are used to derive the IMF orientation from the observed cycloid distributions. One method is intuitive (intuitive method), while the others derive the minimum variance direction of the velocity vectors for the observed ring ions. These velocity vectors are selected either manually (manual method) or automatically using simple filters (automatic method). While the intuitive method and the manual method provide similar IMF orientations by which the observed cycloid distribution is well arranged into a partial circle (representing gyration) and constant parallel velocity, the automatic method failed to arrange the data to the degree of the manual method, yielding about a $30^{\circ}$ offset in the estimated IMF direction. The uncertainty of the derived IMF orientation is strongly affected by the instrument resolution. The source population for
\end{abstract}


these ring distributions is most likely newly ionized hydrogen atoms, which are picked up by the solar wind.

Keywords: IMF, Mars, ion gyration, pick-up process

\section{Introduction}

The European Space Agency Mars Express (MEX) carries the Analyzer of Space Plasma and EneRgetic Atoms (ASPERA-3) experiment (Barabash et al., 2004), which measures hot plasma and energetic neutral atoms (ENA), but MEX does not carry a magnetometer. Without the magnetic field data, it is difficult to interpret plasma processes and ENA formation processes. Therefore, any method by which the magnetic field direction can be obtained aids in interpreting the ASPERA-3 data. In this regard, Fedorov et al. (2006) used the $400 \mathrm{~km}$ circular orbit Mars Global Surveyor (MGS) magnetometer data (Acuna et al., 1998) to derive the interplanetary magnetic field (IMF) direction. Although MGS is not always on the dayside of Mars where the IMF direction can be estimated, MGS produced estimated IMF direction data that is sufficient for large-scale statistics.

Here, we propose an alternative method to utilize the three-dimensional (3-D) ion distribution, based on the work of Mukai and coworkers (Mukai et al., 1986a,b; Terasawa et al., 1986). They derived the direction of the magnetic field in comet Halley's sheath region from ion data obtained by the Suisei spacecraft. The principle takes advantage of the gyration of ions of cometary origin around the IMF. In the solar wind frame, no electric field is imposed on the ions, causing the ions to perform simple spiral motions. In velocity phase space, a spiral motion forms a two-dimensional (2-D) ring trajectory with a constant velocity along the magnetic field. The ring's plane is perpendicular to the local magnetic field. In the actual Suisei data, a 3-D shell-like distribution is observed instead of a 2-D ring, and the orientation of the symmetry axis is considered as parallel to the IMF direction. This principle was also applied to both electron and ion data near the Moon during the flyby of the Nozomi spacecraft (Futaana et al., 2003). In order to obtain the symmetry axis in 3-D velocity space, a measurement of the 3-D proton distribution is required.

The ring distribution is also found in the upstream foot region of the Earth's bow shock, but the source ions are not the newly born ions but the reflected solar wind. Using ISEE-1 and -2 data, Paschmann et al. (1981) and Sckopke et al. (1983) showed that the ion distribution in the upstream foot region of a quasi-perpendicular bow shock is consistent with a partial ring distribution that originates from the reflected solar wind at the bow shock. Later, AMPTE and Cluster observations further demonstrated that the ring distribution exists only within a gyroradius (few hundred km) upstream of the bow shock (Sckopke et al., 1990; Möbius et al., 2001).

For the Martian case, both the newly ionized neutrals and the reflected solar wind can be substantial sources, and therefore, we can expect the ring or shell-like 
distribution to be detected upstream and/or downstream of the bow shock. To obtain the magnetic field direction from the actual MEX data, it is important that the magnetic field direction is nearly uniform over a distance greater than an ion gyroradius and a duration of the observation cycle of 3-D ion distribution measurement. Obviously, the best place for such an attempt is the upstream region of the front-side bow shock, where we actually have observed many cycloid-like ring distributions. It might also be possible to derive the magnetic field direction from the data within the magnetosheath, but this is beyond the scope of this paper.

During 2004 and 2005, the Ion Mass Analyser (IMA) of the ASPERA-3 experiment on board MEX measured the 3-D ion distribution with a nearly 3-min cycle (the operation mode is different in 2006). One 3-D measurement cycle of IMA corresponds to a distance of about $550 \mathrm{~km}$ or one gyroradius for a $1 \mathrm{keV}$ proton in an $8 \mathrm{nT}$ magnetic field. Therefore, IMA is capable of providing data to derive the IMF orientation if the cycloid distribution is observed and if the IMF is constant and uniform during the observation. However, IMA operation is optimized to separate heavy ions (e.g., atomic ions and molecular ions) of Martian origin and the majority of observations were performed in operational modes in which IMA hardly detects ring-distributed protons. Because of this, only one bow-shock crossing in 2004 was observed when IMA was in the appropriate operational mode. In this paper, we use this observation together with one of best observations from 2005 to illustrate the technique of determining the IMF direction from the ring distribution observed by IMA.

\section{Instrument}

The IMA and ELectron Spectrometer (ELS) on board MEX are parts of ASPERA3 experiment (Barabash et al., 2004). ELS has a $4^{\circ} \times 360^{\circ}$ field of view that is divided into 16 azimuthal sectors, each $22.5^{\circ}$ wide. The sensor consists of a top hat electrostatic analyzer in a very compact design. ELS measures electrons in the energy range from $1 \mathrm{eV}$ to $20 \mathrm{keV}$ in logarithmically scaled energy steps every $4 \mathrm{sec}$. For the detail of the ELS instrument, refer to Barabash et al. (2004) and Winningham et al. (2006).

IMA is a top hat instrument that combines an electrostatic energy analyzer with a magnetic mass analyzer. IMA has a $4.6^{\circ} \times 360^{\circ}$ field of view that is divided into 16 azimuthal sectors, each $22.5^{\circ}$ wide. IMA measures ions in the energy range from $10 \mathrm{eV} / \mathrm{q}$ to $30 \mathrm{keV} / \mathrm{q}$ in logarithmically scaled energy steps every $12 \mathrm{sec}$. In order to produce a 3-D particle measurement on the 3-axis stabilized MEX spacecraft, IMA has an electrostatic deflection system (or elevation analyzer) at its entrance, which scans from $-45^{\circ}$ to $+45^{\circ}$ (16 elevations) in approximately 3 min. The actual entrance angle of the ions is slightly energy dependent. The overall field-of-view is approximately $360^{\circ}$ (16 sectors) $\times 90^{\circ}$ ( 16 elevations $)$. 
IMA is primarily designed to examine ions of Martian origin, with an option to sample the solar wind. The mass analyzer (magnets) is designed to deflect the incident solar wind protons away from its position-sensitive detector (microchannel plate or MCP) so that observations do not suffer from contamination by solar wind protons. In order to sample the solar wind with this design, IMA contains an adjustable electrostatic post-acceleration (PA) system between its electrostatic analyzer and magnetic analyzer. With the highest PA voltage, incident solar wind protons are accelerated to fast enough to reach the MCP detector before being deflected significantly by the magnetic mass analyzer. However, this mode was rare during 2004-2005.

IMA has three PA settings: PA $=0$ (nearly no acceleration, about $0.3 \mathrm{kV}$ ), PA $=1($ about $2.4 \mathrm{kV})$, and PA $=2$ (highest acceleration, about $4.2 \mathrm{kV})$. The PA $=0$ mode is optimized to separate heavy ions, and solar wind protons are not detected unless the solar wind is extremely fast. The PA $=2$ mode is optimized to detect the solar wind. The PA $=1$ mode is a marginal mode which detects only a small part of the solar wind (alpha particles and superthermal protons) in most cases. For details of the IMA instrument, refer to Barabash et al. (2004), Lundin et al. (2004), and Fedorov et al. (2006).

\section{Observations}

IMA data from January 2004 through June 2005 were examined for operation in the PA $=2$ mode (including a mode which has an alternating PA for every other full scan). Only one bow shock crossing was identified in 2004, while 38 bow shock crossings were identified during the first half of 2005. The observation from 27 April 2005 near 1337 UT is presented below as one of the best examples observed, followed by the observation from 22 March 2004 near 1230 UT, the first IMA measurement of a bow shock crossing with $\mathrm{PA}=2$.

\subsection{APRIL 2005, 1330 UT}

Figure 1 shows the MEX orbit and energy-time spectrograms of the electron (ELS) and ion (IMA) data during 1331-1357 UT on 27 April 2005. All axes references are made in the Mars-Sun Orbit (MSO) Cartesian coordinate system, with the $+X$ direction pointing sunward, the $+Y$ direction duskward, and the $+Z$ direction toward the north ecliptic pole, and $R^{2}=X^{2}+Y^{2}$. The nearly 3-min $(192 \mathrm{sec})$ cycle seen in the IMA data is due to the scanning cycle of the IMA entrance direction from about $-45^{\circ}$ (elevation $\left.=0\right)$ to about $+45^{\circ}($ elevation $=15)$. Figure 1 contains 7 full scans of IMA data. In the present case, elevation $=0$ corresponds to the northward viewing sector (detecting $45^{\circ}$ southward traveling ions) and elevation $=$ 15 corresponds to the southward viewing sector (detecting $45^{\circ}$ northward traveling 
MEX / ASPERA-3 , 2005-4-27 , 1331-1357 UT
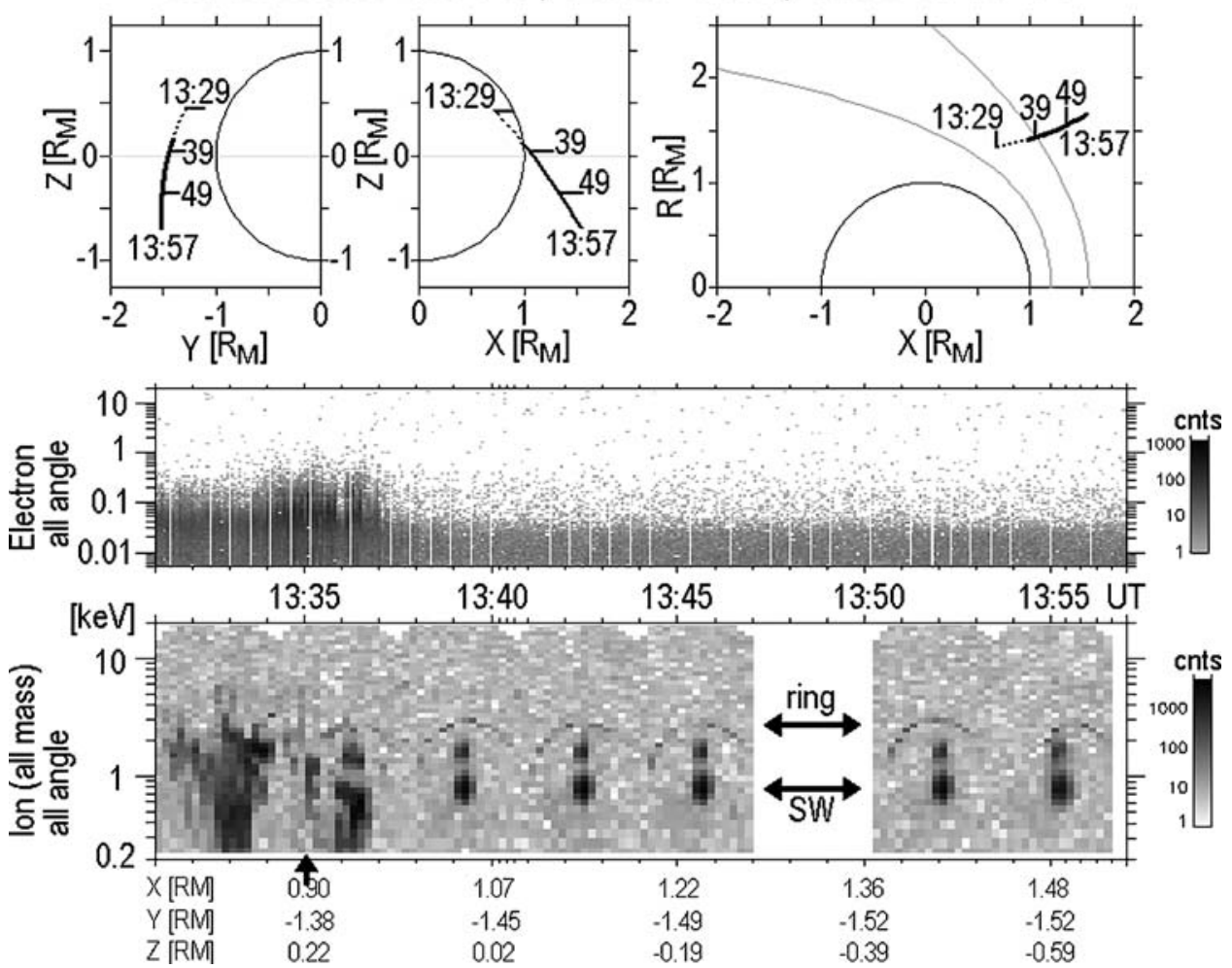

Figure 1. Overview of the MEX orbit and hot plasma data during 1331-1357 UT on 27 April 2005. The upper part shows the MEX orbit in the Mars-Sun Orbit (MSO) Cartesian coordinate system, with the $+X$ direction pointing sunward, the $+Y$ direction duskward, and the $+Z$ direction toward the north ecliptic pole, and $R^{2}=X^{2}+Y^{2}$. The unit " $R_{M}$ " is the Martian radius. The average boundary positions (bow shock and induced magnetosphere boundary) are drawn with grey lines in the upper right panel. The MEX traversal (IMA operational) is drawn by a thick line: the solid line corresponds to MEX outside the bow shock where the partial ring distributions are observed and the dashed line corresponds to MEX inside the bow shock where the magnetosheath-like distributions are observed. The lower panels show the energy-time spectrograms of electrons (from ELS, $5 \mathrm{eV}-20 \mathrm{keV}$ ) and ions (from IMA, $0.2-20 \mathrm{keV}$ ). All mass and azimuthal angles are integrated. The nearly 3-min cycle seen in the IMA data is due to the electric scan of the entrance direction from nearly $-45^{\circ}$ (elevation $=$ 0 ) to nearly $+45^{\circ}$ (elevation $=15$ ). From both ELS and IMA, the bow shock outbound is identified at around 1337 UT. Horizontal arrows in the IMA data indicate the cycloidal ions (see text) and the solar wind protons, and vertical arrow at the bottom (at around 1335 UT) indicates a high count rate discussed at the end of Section 3.1.

ions), while azimuthal sector 2 is pointing toward $+X$ (detecting tailward traveling ions), azimuth $=14$ is pointing toward $+Y$ (detecting ions traveling toward the $-Y$ direction), and azimuth $=6$ is pointing toward $-Y$ (detecting ions traveling toward the $+Y$ direction) in the MSO coordinates. 
The spacecraft traversed the bow shock on the dawn side (outbound) at around 1337 UT, as identified by the sudden change of the energization/thermalization level of the solar wind as observed by both IMA and ELS. During the next 20 min (5 full elevation scans of IMA), IMA detected a partial ring-like distribution of ions at around $2-3 \mathrm{keV}$ (indicated by the upper horizontal arrow shown in Figure 1), well above the solar wind alpha particles $(\sim 1.6 \mathrm{keV})$ and the solar wind protons $(\sim 0.8 \mathrm{keV}$, indicated by the lower horizontal arrow shown in Figure 1). Since the time axis is the same as the elevation scan within each 3-min scanning cycle, this ring-like structure actually means that the velocity depends on the direction as one would expect with a partial ring-like distribution in velocity space.

Figure 2 shows the energy-time spectrograms of IMA organized by the masscharge ratio (protons in the lower half and alpha particles in the upper half) and by azimuthal sectors (in individual panels) during 1334-1344 UT, i.e., covering the $2 \mathrm{nd}$, 3rd, and 4th full scans of Figure 1. The mass channel selection can be confirmed by Figure 3, which shows the energy-mass matrix during two full scans (1337-1344 UT). At 1339:20 UT and 1342:30 UT in Figure 2, the solar wind is clearly separated into protons at around $0.8 \mathrm{keV}$ and alpha particles at around 1.6 $\mathrm{keV}$, and they are registered at azimuthal scan 2 and elevation scan 9 (closer to scan 8 than scan 10). The separation between alpha particles and protons is clearer in Figure 3 (lower middle panel). Note that solar wind protons strongly contaminate all mass channels (contamination is observed at all mass channels at around 0.8 $\mathrm{keV}$ in Figure 3).

The partial ring distribution is recognized during all 3 full scans in Figure 2, and is detected at a single azimuthal sector (azimuth $=3$ ) for a wide range of elevation angles (from elevation $=2$ at 1341:00 UT to elevation $=15$ at 1343:40 UT for the third full scan, and from elevation $=4$ at 1338:10 UT to elevation $=15$ at 1340:30 UT for the second full scan). The counts at elevation $=3 /$ azimuth $=2$ at 1338:00 UT during the second full scan are also connected to the ring, but no other counts are found at azimuth $=2$ or azimuth $=4$ in conjunction with the ring at azimuth $=3$. Thus, these ions are distributed in a 2-D plane rather than in a 3-D shell. Its direction (azimuth $=3$ ) is one sector $\left(22.5^{\circ}\right)$ off from the solar wind direction. In Figure 2, the ring distribution is recognized in the proton channel only, and its composition is confirmed from the energy mass matrix shown in Figure 3 (upper three panels).

For the purpose of further analysis, we list the energy and direction of this ring distribution during the full scan of 1344-1347 UT in Table I. The first three columns are elevation scans (El), azimuthal sectors (Az), and the corresponding viewing directions (unit vector components) in the MSO coordinates (see Figure 1 for the $X, Y$, and $Z$ directions). The center energy (keV) of the ring distribution is listed in the next column, and this energy is converted into the velocity components $(\mathrm{km} / \mathrm{s})$ using MSO coordinates (the last three columns). The information in Table I is basically enough to derive the IMF orientation. 

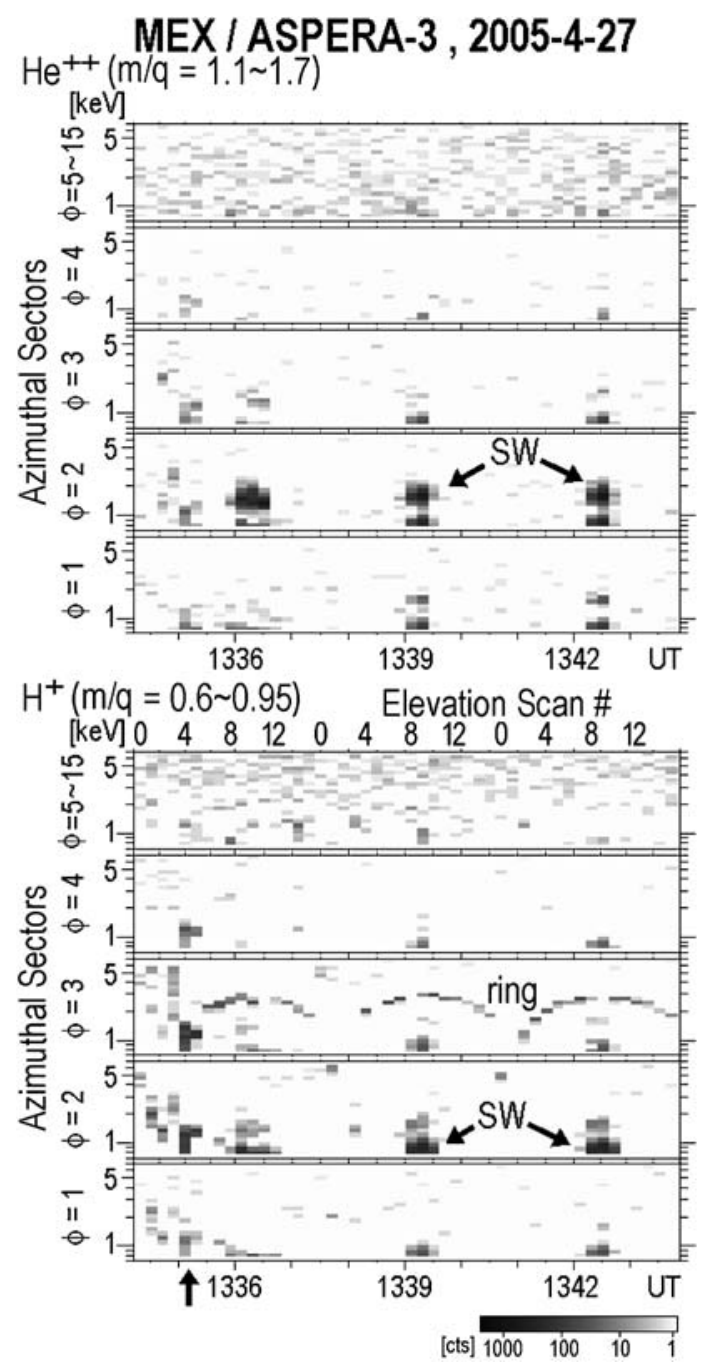

Figure 2. Energy-time spectrograms of light ions between 0.7 and $7 \mathrm{keV}$ during 1334:00-1343:40 UT on 27 April 2005. Five different azimuthal sectors $(\phi)$ are presented for both the proton masschannel (lower 5 panels) and the alpha particle mass-channel (upper 5 panels). These 5 azimuthal sectors are: 1, 2, 3, 4, and the integration of 5 through 15 . Sector 0 is not presented because it gathers contamination from all of the other sectors. Note that IMA scans 16 elevation directions in nearly 3 min, and the presented period corresponds to 3 complete scans, with the first pixel on the time axis corresponding to elevation $=0$ (about $-45^{\circ}$ at $2 \mathrm{keV}$ ) and the last pixel on the time axis corresponding to elevation $=15$ (about $+40^{\circ}$ at $2 \mathrm{keV}$ ) as indicated at the top of proton panel. The solar wind is seen at elevation $=9$ (weak at elevation $=8$ ). The counts that are seen above the solar wind in the proton channel are due to contamination from alpha particles and the counts that are seen below the solar wind in the alpha particle channel are due to contamination from protons. The vertical arrow at the bottom indicates the intense count that is shown in Figure 1. 


\section{TABLE I}

Direction and energy of the registered ring during 1344-1347 UT, 27 April 2005.

\begin{tabular}{lllllc}
\hline El Az & Sensor direction $(X, Y, Z)$ & $E(\mathrm{keV})$ & $V_{X}(\mathrm{~km} / \mathrm{s})$ & $V_{Y}(\mathrm{~km} / \mathrm{s})$ & $V_{Z}(\mathrm{~km} / \mathrm{s})$ \\
\hline 0203 & $(0.81,-0.29,0.51)$ & 1.37 & -414 & 148 & -260 \\
0303 & $(0.85,-0.30,0.42)$ & 1.86 & -508 & 181 & -251 \\
0403 & $(0.89,-0.32,0.33)$ & 2.27 & -584 & 208 & -218 \\
0503 & $(0.91,-0.33,0.24)$ & 2.49 & -630 & 225 & -164 \\
0803 & $(0.94,-0.34,-0.05)$ & 2.74 & -680 & 243 & 39 \\
0903 & $(0.93,-0.33,-0.15)$ & 2.74 & -673 & 241 & 110 \\
1003 & $(0.91,-0.33,-0.25)$ & 2.74 & -659 & 236 & 180 \\
1203 & $(0.85,-0.30,-0.43)$ & 2.49 & -585 & 210 & 298 \\
1303 & $(0.80,-0.29,-0.52)$ & 2.27 & -529 & 190 & 341 \\
1403 & $(0.75,-0.27,-0.60)$ & 2.00 & -464 & 167 & 370 \\
1503 & $(0.69,-0.25,-0.68)$ & 1.68 & -392 & 141 & 382 \\
\hline
\end{tabular}

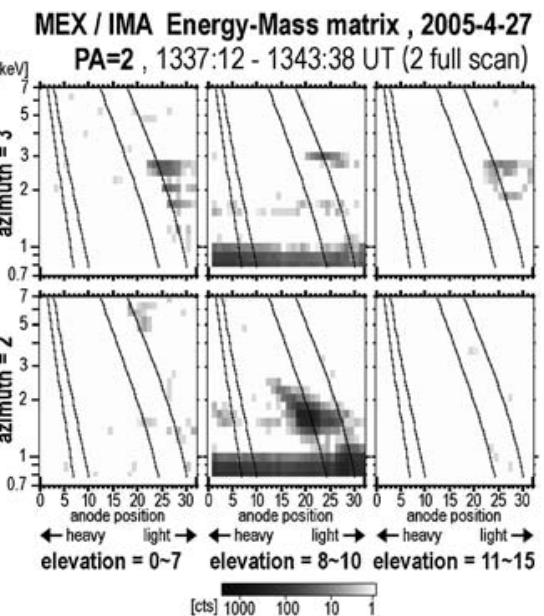

Figure 3. Energy-Mass matrices during 2 full scans (1337:10-1343:40 UT) of Figure 2. The horizontal axis of each panel is the detector position that corresponds to a different mass value for each given energy. The curved lines in each panel correspond to (from right to left) mass per charge $m / q$ $=1, m / q=2, m / q=16$, and $m / q=32$. The upper 3 panels show data from azimuthal sector 3 where the partial ring distribution is found in Figure 2 and the lower 3 panels show data from the azimuthal sector 2 where the solar wind is detected for elevations $=8-10$. In the left, middle, and the right panels, the counts are integrated over elevation $=0-7\left(-45^{\circ}\right.$ to $\left.0^{\circ}\right)$, elevation $8-10\left(0^{\circ}\right.$ to $\left.+15^{\circ}\right)$, and elevation $11-15\left(+15^{\circ}\right.$ to $\left.+45^{\circ}\right)$, respectively. Note that solar wind protons with an energy of about $0.7-1 \mathrm{keV}$ strongly contaminate all mass channels due to a mode-dependent instrumental effect (marginally deflected protons by the magnetic mass analyzer to hit the outer boundary of the instrument near the MCP, and scatter randomly). 

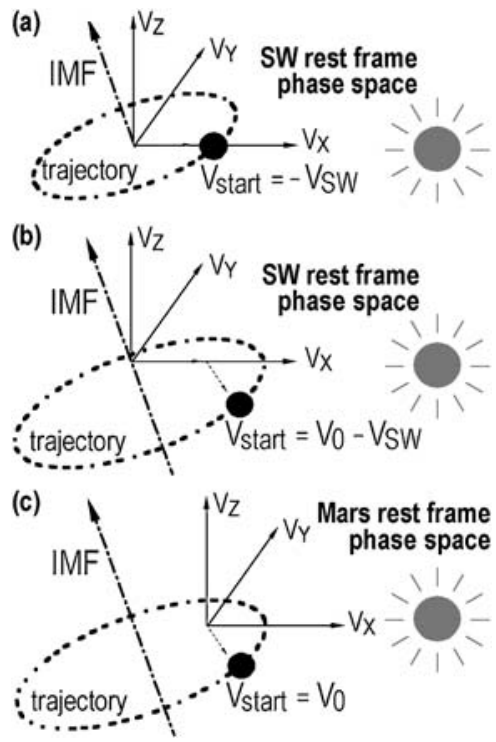

Figure 4. Illustration of ion motion in the solar wind $\left(\mathbf{V}_{\mathrm{SW}}\right)$ with respect to the IMF in a velocity space diagram $\left(V_{X}, V_{Y}, X_{Z}\right)$ where $X, Y, Z$ directions are given in Figure 1. (a) An ion with zero initial speed (i.e., negligible compared to the solar wind velocity) in the Martian rest frame has an initial velocity of $-\mathbf{V}_{\mathrm{SW}}$ in the solar wind frame. Since there is no electric field in the solar wind frame, the ion performs a simple spiral motion around the IMF (the magnetic field direction is about the same in both the Martian rest frame and the solar wind frame). Any spiral motion is represented by a circle about the magnetic field in velocity space. (b) For the finite initial velocity $\left(\mathbf{V}_{0}\right)$ in the Martian rest frame, the start position is shifted by $\mathbf{V}_{0}$ from (a), and hence the radius of the ring changes. However, the ring is still found around the IMF and the ion speed (distance from the origin of coordinates) is constant. (c) The Martian rest frame trajectory of what is shown in (b). The difference between (b) and (c) is only a constant velocity $+\mathbf{V}_{\text {SW }}$.

To understand the relation between the ring-like distribution and the IMF orientation, we illustrate velocity space ion motion $\left(V_{X}, V_{Y}, V_{Z}\right)$ in the solar wind in Figure 4. Note that the magnetic field is almost the same (non-relativistic limit) between the Martian rest frame and the solar wind frame. We first consider a newly ionized neutral atom with nearly zero initial velocity in the Martian rest frame. Such an ion has an initial velocity of $-\mathbf{V}_{\text {SW }}$ (where $\mathbf{V}_{\text {SW }}$ is the solar wind velocity) in the solar wind frame as illustrated in Figure 4a.

Since there is no electric field in the solar wind frame, any ion with a velocity different from the solar wind performs a simple spiral motion (circular motion around the magnetic field plus constant motion along the magnetic field) with a constant speed in the solar wind frame. The spiral motion of an ion is represented by a ring trajectory which is symmetric about the magnetic field in velocity space. The constant speed in velocity space means that the symmetry axis of the trajectory (i.e., magnetic field) passes through the origin in the solar wind frame. Figure $4 \mathrm{~b}$ illustrates the general case of a finite initial velocity $\mathbf{V}_{0}$ in the Martian rest frame 
(initial velocity $\mathbf{V}_{0}-\mathbf{V}_{\mathrm{SW}}$ in the solar wind frame). While the start position and radius of the ring in velocity space are different from the previous case (Figure 4a), the orientation of the ring is still the same as that in Figure 4a; i.e., the ring plane is again perpendicular to the magnetic field, and the symmetry axis (magnetic field) again passes through the origin of coordinates in the solar wind frame.

Strictly speaking, feedback of ring ions to the magnetic field (e.g., diamagnetic effect) may deforms the IMF orientation if the mass flux of these ring ions is significant. Such deformation is indeed important near the ionopause or induced magnetosphere boundary. However, the mass flux of the ring component is much lower than that of the solar wind (Figures 1 and 2), and the orientations of the rings are nearly the same for many full elevation scans. Thus, the feedback from the ring ions to the IMF orientation is ignorable inside the solar wind, and the above test particle approximation is appropreate in deriving the IMF orientation.

Figure $4 \mathrm{c}$ illustrates the same ion motion as Figure $4 \mathrm{~b}$ in the Martian rest frame, i.e., with initial velocity $\mathbf{V}_{0}$. Although what the particle instrument detects is the energy seen in the spacecraft rest frame, the spacecraft velocity relative to Mars is negligible compared to the solar wind velocity for the MEX observations. The Lorenz transform in velocity space from the solar wind frame to the Martian rest frame means an addition of the constant velocity $\mathbf{V}_{\mathrm{SW}}$ to the ring trajectory illustrated in Figure $4 \mathrm{~b}$. Therefore, the radius and orientation of the ring is the same between Figure $4 \mathrm{~b}$ and Figure $4 \mathrm{c}$; i.e., the ring plane in velocity space is always perpendicular to the IMF in both rest frames. The resulting ring trajectory in velocity space does not necessarily have its symmetry axis (IMF axis) aligned to any of $V_{X}, V_{Y}$, or, $V_{Z}$ axes, but this symmetry axis always intersects the $V_{Y}-V_{Z}$ plane in velocity space at $\left(V_{X}, V_{Y}, V_{Z}\right)=\left(-V_{\mathrm{SW}}, 0,0\right)$.

The motion depicted in Figure 4c can also be seen as the motion of an arbitrary ion under a constant IMF and a constant solar wind electric field $\mathbf{E}_{\mathrm{SW}}=-\mathbf{V}_{\mathrm{SW}} \times \mathbf{B}$ in the Martian rest frame (where $\mathbf{B}$ is the IMF vector). Since $\mathbf{E}_{\mathrm{SW}}$ does not have any parallel component to $\mathbf{B}$, the acceleration direction of the ion is always perpendicular to the IMF, making the velocity space trajectory stay within a plane (a ring under constant fields) perpendicular to the IMF. This $\mathrm{E} \times B$ drift has an average velocity in the Martian rest frame of $\mathbf{E}_{\mathrm{SW}} \times \mathbf{B} / B^{2}=\left(\mathbf{V}_{\mathrm{SW}}\right)_{\perp}$ for the velocity component perpendicular to the IMF and $\left(\mathbf{V}_{0}\right)_{/ /}$for the velocity component parallel to the IMF.

Since the location of the symmetry axis depends only on the solar wind velocity and the IMF orientation, the velocity space trajectory is the same for different IMF strengths as long as the IMF orientation is the same. For example, if $\mathbf{V}_{0}=0$ (e.g., pick up of newly ionized hydrogen corona by the solar wind), the trajectory in velocity space is a circle passing through the origin in Figure 4c. The ion trajectory in velocity space thus reflects the IMF orientation and the initial velocity of the ion, but not the strength of the IMF.

In observations, we deal with an ensemble of ions with different initial velocities, and in this case, the velocity space trajectories of these ions are not necessarily the same. Yet, if the observed distribution is symmetric around an axis, this axis is 
most likely aligned to the magnetic field direction. Mukai et al. (1986b) used this information to derive the magnetic field direction from a shell-like distribution observed by the Suisei spacecraft.

Unlike in the comet observations of Mukai et al. (1986b), IMA detected a 2$\mathrm{D}$ ring distribution. It is very difficult for all the trajectories to lie within a plane unless the spread of the initial velocity (thermal velocity) is much smaller than the solar wind velocity (about $400 \mathrm{~km} / \mathrm{s}$ in the present case). A small spread in the initial velocity means that the original ion distribution in the Martian rest frame must be either beam-like or of nearly zero velocity (e.g., newly ionized hydrogen corona) upstream of the satellite for about a proton gyroradius. In other words, the responsible source ions must be either a stable beam (duration more than the observation time, i.e., more than $20 \mathrm{~min}$ ) or at nearly zero velocity, both for a wide region (more than one proton gyroradius) upstream of the satellite location.

In both cases, the IMF direction must be perpendicular to the plane occupied by the ring distribution (we hereafter call it a "ring's plane"). In the present case, the IMF is perpendicular to the azimuth $=3$ meridian. The attitude of the spacecraft is such that azimuth $=3$ corresponds to a meridian plane (perpendicular to the $X-Y$ plane) lying about $\pi / 8$ westward of the $X-Z$ plane. Therefore, the IMF is lying nearly in the $X-Y$ plane $\left(B_{Z} \ll B\right)$ with $B_{X} / B_{Y} \sim \tan (\pi / 8)$.

There is an ambiguity with the sign; i.e., we do not know if $B_{Y}>0$ or $B_{Y}<0$. Determining the sign of IMF orientation is not easy. Although the ions perform right-handed gyro orbits in both real space and velocity space, this knowledge helps very little in finding out which way the velocity vectors of the observed gyrating ions have evolved along the ring trajectory (e.g., clockwise or counter-clockwise in Figure 4 when looking from $+Z$ ). The Table I data does not give information whether the ring ions evolve from elevation $=2$ to elevation $=15$ (corresponding to $\left.B_{Y}<0\right)$ or elevation $=15$ to elevation $=2\left(\right.$ corresponding to $\left.B_{Y}>0\right)$.

In Table I, the ring distribution reaches the maximum speed at azimuth $=3 / \mathrm{el}$ evation $=9$. This direction is only one azimuthal sector away (the same elevation scan) from the solar wind direction (or $-V_{X}$ axis). This tilt angle (about $\pi / 8$ ) is the same as the angle between the $Y-Z$ plane and the IMF direction derived above. This is not a coincidence. In Figure $4 \mathrm{c}$, for small $\mathbf{V}_{0}$, the angle between the direction of the maximum speed and the $-V_{X}$ direction is the same as the angle between the IMF direction and the $Y-Z$ plane. Therefore, if this angle is small, $B_{X}$ is most likely small compared to $B_{Y}$ or $B_{Z}$.

Note that the observed ring distribution covers only a part of the expected circle because IMA can only detect protons with energies higher than few hundred $\mathrm{eV}$, even in the operational mode designed to observe protons $(\mathrm{PA}=2$ mode). As shown in Figure $4 \mathrm{c}$, the ring distribution in velocity space is shifted by the solar wind velocity, making its energy in the spacecraft coordinate high in the $-V_{X}$ direction and low in the $\mathrm{V}_{X}$ direction. Therefore, an instrument with a limited energy coverage can only detect a part of the total ring distribution (more than a certain distance away from the origin in velocity space (see Figure 4c)). For example, we can detect the 
ring distribution in only one of two oppositely looking sectors. For the present case, we did not observe the ring distribution at azimuthal sector 11 that is $180^{\circ}$ away from sector 3. No outstanding signature is recognized in the top and 6th panels of Figure 2, which show the integrated ion counts for azimuthal sectors 5-15.

The ring's plane (or its normal direction) can also be obtained from the minimum variance method (Sonnerup and Cahill, 1967; see also Sonnerup and Scheible, 1998) by applying it to the registered velocities of the ring distribution if the data set is clean (i.e., composed of the gyrating component only). This method determines the direction in which the data scatter the least. This minimum variance direction is represented by an eigenvector for the minimum eigenvalue of a matrix calculated from the data.

One major problem with using the minimum variance method is the cleaning of the data (to choose only the ring data) because the ring distribution is a minor population compared to the solar wind. The solar wind counts severely deform the minimum variance direction. Furthermore, there are other counts that do not belong to either the ring or the solar wind. Extracting only the ring population (which is just one of the secondary populations) from the data is not a simple task. The most reliable way is to visually-identify the ring population and construct a reliable set of data. Table I is an example of such a manually constructed data. The minimum variance direction obtained from the velocity data of Table I is $(b x, b y, b z)= \pm(0.34,0.94,-0.003)$ in MSO coordinates. The intuitive method agrees well with the minimum variance result on this manually selected data.

It would be ideal if one could automatically filter the raw data to construct a set composed only of the ring component. We have tried various filters, such as a count filter (optimized filter is $7 \leq$ count $\leq 40$ for the present case) and an energy filter (optimized filter is $K_{\mathrm{SW}} / 4<K_{\mathrm{REF}}<1.5^{*} K_{\mathrm{SW}}$ for the present case, where $K_{\mathrm{REF}}$ is the kinetic energy of the ion in the solar wind frame and $K_{\mathrm{SW}}$ is the solar wind energy).

Some of the results using various filter thresholds together with the results from the manual method are listed in Table II. The first column describes the method used to obtain the ring data. The square root of the maximum, medium, and minimum eigenvalues (in $\mathrm{km} / \mathrm{s}$ ) are listed in the next three columns, followed by the minimum variance directions (unit vector components) in MSO coordinates. The angle between the derived minimum variance direction and that obtained from Table I (they are given in first two rows in Table II) are listed in the last two columns.

The automatic filter removes the majority of the solar wind; however, this is not enough. The minimum variance direction for the automatically filtered 1337:00-1357:00 UT data with the optimum threshold is $(b x, b y, b z)=$ $\pm(-0.20,0.95,-0.23)$ in MSO coordinates, which is $34^{\circ}$ off from the manual method result. To illustrate the difference, we plot the observed velocities in the three different coordinates in Figure 5.

Figure 5 shows the velocity scatter plots in (a) MSO (XYZ) coordinates, (b) local Cartesian (LMN) coordinates determined by the minimum variance method which 
TABLE II

Minimum variance analyses for various data sets.

\begin{tabular}{lccclll}
\hline Data set & $\mathrm{L}_{L}{ }^{*}$ & $\mathrm{~L}_{M}{ }^{*}$ & $\mathrm{~L}_{N}{ }^{*}$ & B direction $(b x, b y, b z)$ & offset $^{* *}$ & offset $^{* *}$ \\
\hline Table I & 248.5 & 102.9 & \multicolumn{1}{c}{0} & $\pm(0.337,0.942,-0.003)$ & - & $11.8^{\circ}$ \\
Table I I** & 255.8 & 113.2 & 37.5 & $\pm(0.516,0.854,-0.062)$ & $11.8^{\circ}$ & - \\
$7 \leq$ count $\leq 40$ & 203.3 & 153.7 & 77.7 & $\pm(-0.195,0.953,-0.232)$ & $33.7^{\circ}$ & $43.3^{\circ}$ \\
$7 \leq$ count $\leq 40^{* * * *}$ & 236.5 & 188.8 & 130.2 & $\pm(0.733,0.661,-0.161)$ & $29.5^{\circ}$ & $17.7^{\circ}$ \\
$5 \leq$ count $\leq 25$ & 202.3 & 149.6 & 80.6 & $\pm(-0.259,0.958,-0.120)$ & $35.4^{\circ}$ & $46.2^{\circ}$ \\
$10 \leq$ count $\leq 50$ & 205.5 & 162.7 & 62.5 & $\pm(-0.272,0.909,-0.315)$ & $40.1^{\circ}$ & $49.0^{\circ}$ \\
\hline
\end{tabular}

* Square root of the eigenvalues (in $\mathrm{km} / \mathrm{s}$ ).

**Angle from minimum variance direction obtained from Table I.

***Add one data point (elevation $=2 /$ azimuth $=2$ at $1371 \mathrm{eV}$ at $1334 \mathrm{UT}$ ) to Table I (see Section 4.2).

****From 1334-1357 UT data. Otherwise, from 1337-1357 UT data (see Section 4.2).

is applied to Table I data (manual method), and (c) LMN coordinates determined from automatically filtered data (automatic method). The LMN coordinates are defined by the maximum variance direction $(\mathbf{L})$ and the minimum variance direction (N) (Sonnerup and Cahill, 1967).

One can recognize that the variance in the $\mathbf{V}_{N}$ direction (horizontal alignment of the data in the $L-N$ projection and $M-N$ projection) is zero in Figure $5 \mathrm{~b}$ but not in Figure 5c. The zero variance in the $\mathbf{V}_{N}$ direction means that the velocity is constant in the $\mathbf{V}_{N}$ direction, i.e., ions receive no electromagnetic force in this direction. The $L-M$ projection plot is closer to a circle in Figure $5 \mathrm{~b}$ than in Figure $5 \mathrm{c}$, and the center of the circle is located where many non-ring data (representing the solar wind) are clustered. The good fit to a circle means that the observed ring distribution is composed of gyrating ions around the IMF with the same initial velocity, and that the center of the circle represents the effective solar wind velocity by which the ring ion is exposed to the convection $\left(-\mathbf{V}_{\mathrm{SW}} \times \mathbf{B}\right)$ electric field in the Martian rest frame.

These facts mean that the LMN coordinates in Figure $5 b$ (with $\mathbf{N}$ direction $=(0.34,0.94,-0.003))$ determine the ring's plane much better than the LMN coordinates in Figure $5 \mathrm{c}$ (with $\mathbf{N}$ direction $=(-0.22,0.96,-0.18)$ ). Thus, the IMF orientation should be estimated from the $\mathbf{N}$ direction of Figure $5 \mathrm{~b}$, but not of Figure 5c. Furthermore, all ring data during 1334:00-1357:00 UT nearly lie on the same circle. This means that the ring's orientation and diameter (i.e., the IMF orientation and the solar wind velocity component perpendicular to the IMF) did not change very much during these 6 full scans. The estimated IMF, which is nearly uniform and constant during the observation, is pointing about $20^{\circ}$ off from the $+Y$ direction toward the $+X$ direction for the 27 April 2005 event. The automatic method gives the IMF direction about $30^{\circ}$ off from this best estimate. 

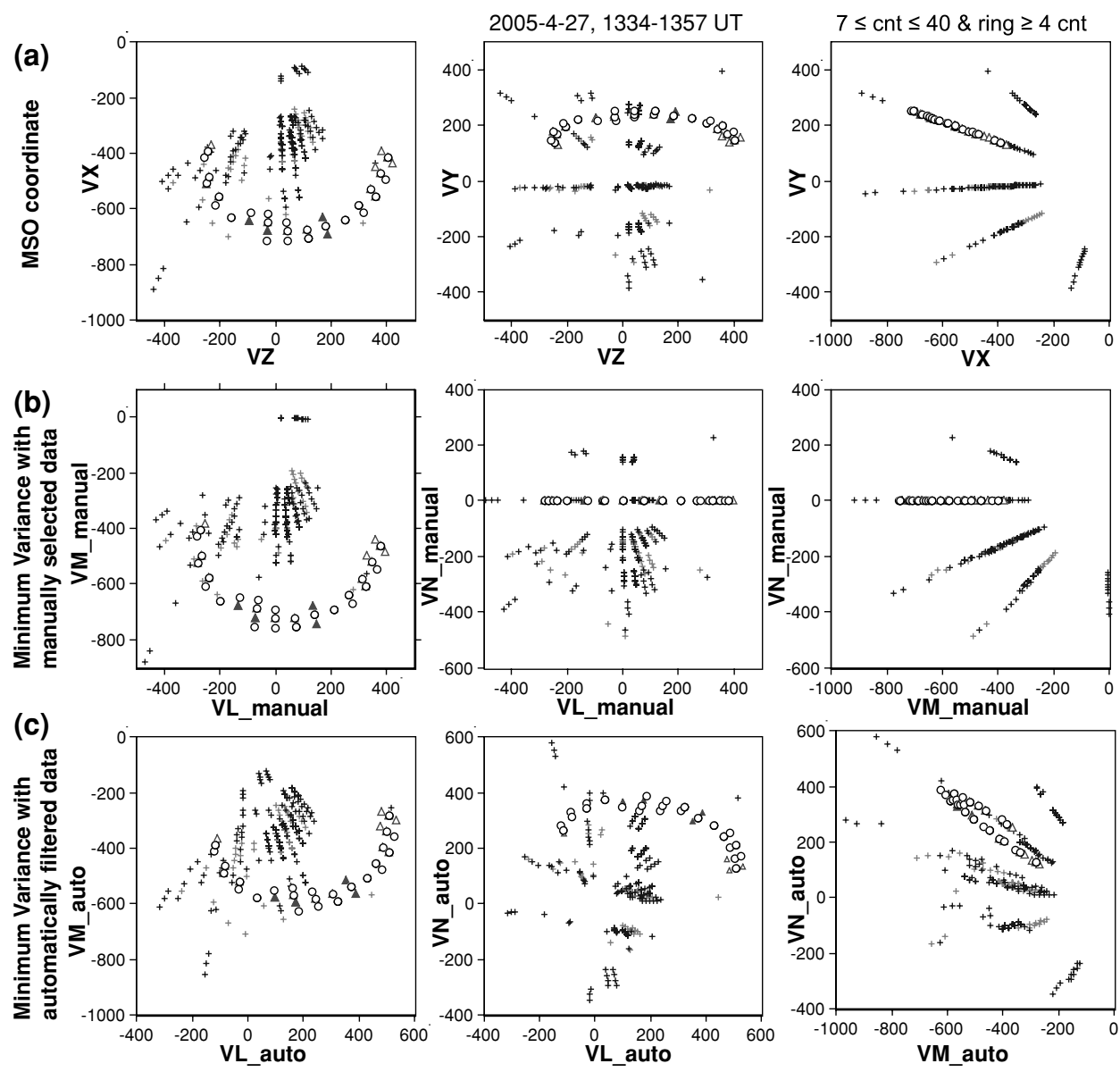

- ring $(7 \leq \mathrm{cnt} \leq 40)$
$\triangle$ ring $(40<\mathrm{cnt}) \quad \Delta$ ring $(4 \leq \mathrm{cnt} \leq 6)$

+ others $(7 \leq \mathrm{cnt} \leq 40)$

others $(40<\mathrm{cnt})$

Figure 5. Velocity scatter plots of the ion data (all axes are in km/s) during 1334-1357 UT, 27 April 2005, in (a) MSO coordinates, (b) local LMN (minimum variance) coordinates determined from Table I (manual method) and (c) local LMN coordinates determined from automatically filtered data during 1337-1357 UT. The selected data are ion mass range 0.5-1.1 (proton channel: it is slightly contaminated by the solar wind alpha particles as seen in Figure 2). Plotted data consist of points that have more than 3 counts and azimuth $=0$ data are not shown (total 450 points). The circles and triangles denote to the point belong to the ring distribution by a manual inspection, with circle corresponding to $7-40$ counts, filled triangle corresponding to more than 40 counts, and empty triangle corresponding to 4-6 counts. Non-ring data are shown using plus marks (black: count is $7-40$, grey: count more than 40).

For the second and the third full scans in Figure 2, the start direction of the ring distribution is close to the direction in which a high count rate is observed at 1335:00 UT (azimuth $=2-3$ and elevation $=4-5$ as indicated by a vertical arrow at the bottom of Figures 1 and 2) with exactly the same energy as the ring distribution 
(around $1.3 \mathrm{keV}$ at azimuth $=3 /$ elevation $=4-5$ ). Although we cannot distinguish whether this high count rate at 1335:00 UT represents a spatial structure (i.e., beam) or a temporal structure (i.e., narrow boundary) from a single spacecraft, the perfect match in directions between this high count rate and the ring distribution suggests that they could be related. The peak count rate is found at the velocity $(-440 \mathrm{~km} / \mathrm{s}$, $-20 \mathrm{~km} / \mathrm{s}, 260 \mathrm{~km} / \mathrm{s}$ ) in MSO coordinates, i.e., at exactly the same velocity as the solar wind but with a substantial $Z$ component.

Since the ring distribution covers nearly half a circle in velocity space, the registered counts of the ring distribution provides nearly half the ring distribution flux. The total number of counts registered for the ring distribution is about 250-300 counts/scan (nearly constant during 1337-1357 UT, i.e., nearly the same for all full scans) in the energy range of 2-3 keV. Meanwhile, solar wind alpha particles are registered with a count rate of about 2000 counts/scan (again, nearly constant) at an energy of about $1.6 \mathrm{keV}$. Since the count rate is roughly proportional to the energy flux, the total mass flux of the ring distribution (entire ring circle) is about $20 \%$ of the solar wind alpha particle flux. As mentioned in Section 2, we cannot get the total proton flux because of the instrumental limit at low energies (Fedorov et al., 2006). The registered solar wind proton count rate (we took the mass range to be $m / q=0.5-6.0$ at energy $0.6-1.0 \mathrm{keV}$ ) is about 5000 counts/scan (again, nearly constant), setting an upper limit to the alpha/proton mass flux ratio of about $10 \%$. Combined, the flux of the ring distribution is less than $2 \%$ of the solar wind flux.

\subsection{MARCH 2004, 1230 UT}

The previous example is an ideal case because the ring distribution is detected in a single azimuthal sector over many elevation scans. However, the ring distribution is generally registered over different azimuthal sectors. Here, we present one such case. Figure 6 shows the MEX orbit and energy-time spectrograms of electrons and ions during 1223-1240 UT on 22 March 2004 (5 full scans). This is the only bow-shock crossing with PA $=2$ mode (optimized for proton detection) during 2004.

The spacecraft traversed the bow shock (outbound) at around 1231 UT (end of the ramp) to 1240 UT (end of the foot): the ion data shows nearly undisturbed solar wind after 1231 UT while the electron data shows an extended foot (slightly heated from the solar wind) until about 1240 UT. The partial ring-like distribution is seen during the last full elevation scan at 1237-1240 UT (indicated by the upper horizontal arrow), well above the solar wind ( $1 \mathrm{keV}$ for protons, indicated by the lower horizontal arrow). The highest energy portion of the ring is also recognized in the previous two full scans at around $4 \mathrm{keV}$.

Figure 7 shows the energy-time spectrograms from IMA, separated into protons and alpha particles at different azimuthal sectors at 1230-1240 UT, i.e., during the last 3 full scans of Figure 6 . The solar wind is detected at elevation $=8$ /azimuth $=$ 


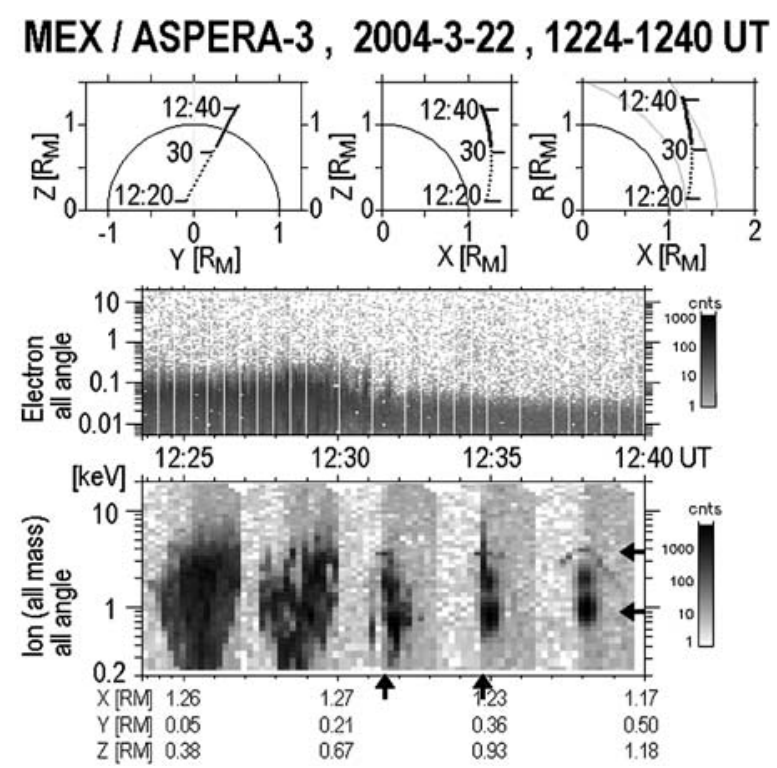

Figure 6. Overview of the MEX orbit and hot plasma data during 1223-1240 UT on 22 March 2004. The format is the same as Figure 1. ELS data indicates the bow shock outbound at around 1240 UT (end of the foot), while IMA data show nearly undisturbed solar wind already after 1231 UT (end of the ramp).

3 with a proton energy of about $1 \mathrm{keV}$ and an alpha particle energy of about $2 \mathrm{keV}$. The ring distribution during the last full scan at 1237-1240 UT consists of protons in the energy range between 2 to $4 \mathrm{keV}$. The energy and direction of the identified ring distribution during the last full scan is summarized in Table III (same format as Table I).

The ring is again distributed in a plane rather than on a 3-D shell, passing exactly through the solar wind direction (elevation $=8 /$ azimuth $=3$ ) at its highest energy of $4 \mathrm{keV}$ (or about $900 \mathrm{~km} / \mathrm{s}$ ) that corresponds to twice the solar wind velocity. In the first 2 full scans, only the highest energy portion is visible at around 1231:30 UT (elevation $=6-7 /$ azimuth $=3$ ) and 1234:50 UT (elevation $=8$ /azimuth $=3$ ), although the rings' directions of these 2 full scans are not clear due to the bow shock crossing.

As mentioned in Section 3.1, the matching of directions between the solar wind and the ring's highest energy suggests that the IMF $B_{X}$ is small compared to the other components. Therefore, the IMF direction inferred from this ring plane lies on a plane that azimuth $=7$ (or 15) covers, and is tilted more than $45^{\circ}$ from the center elevation direction (elevation $=8$ ) of azimuth $=7$ (or 15). Since this center elevation direction is nearly in the $Z$ direction, the IMF direction is estimated as lying almost within the $Y-Z$ plane and with $B_{Z} / B_{Y} \leq 1$. Note that for such an intuitive derivation, we have to compare three directions in velocity space, one at 


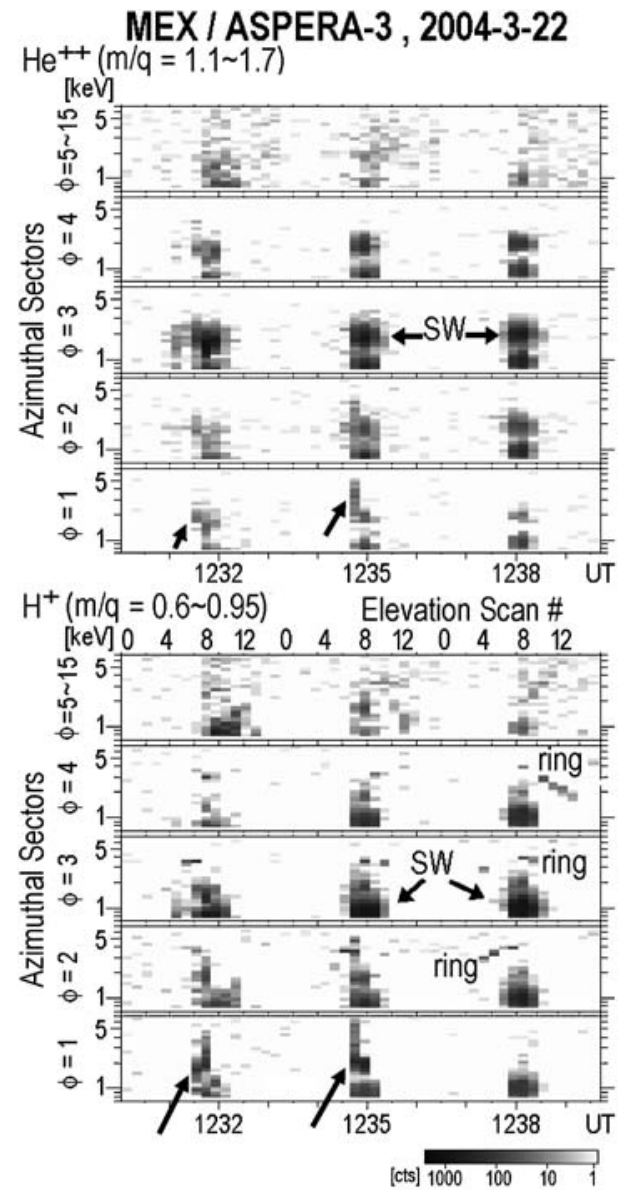

Figure 7. Energy-time spectrograms of light ions between the energies of 0.7 and $7 \mathrm{keV}$ during 1230 1240 UT on 22 March 2004. The format is the same as Figure 2, except that there is no data between elevations 2 through 6 of azimuth $=1$. Also, the quadrant of azimuth 9 through 15 and elevation 0 through 7 is blocked for this particular event.

the highest energy, and the others at the same (lowest possible) energy at both sides relative to the viewing direction that registers the highest energy of the ring.

To confirm this intuitive result, we again employ the minimum variance method. The minimum variance direction obtained from the velocity data shown in Table III is $\pm(-0.01,0.88,0.47)$ in MSO coordinates; i.e., the IMF orientation for the 22 March 2004 event is estimated as $B_{X} \ll|B|$ and $B_{Z} / B_{Y} \sim+0.5$ (lying almost within the $Y-Z$ plane and tilted nearly $30^{\circ}$ from the $Y$ axis). The intuitive method again agrees with the minimum variance result from the manually selected data. The velocity scatter plots in both the MSO coordinates and the local Cartesian LMN coordinates determined by the minimum variance method are shown in Figure 8 (the same format as Figure 5). The scatter in the $\mathbf{V}_{N}$ direction is due to the $\pi / 8$ 
TABLE III

Direction and energy of the registered ring during 1237-1239 UT, 22 March 2004.

\begin{tabular}{lllllc}
\hline El Az & Sensor direction $(X, Y, Z)$ & $E(\mathrm{keV})$ & $V_{X}(\mathrm{~km} / \mathrm{s})$ & $V_{Y}(\mathrm{~km} / \mathrm{s})$ & $V_{Z}(\mathrm{~km} / \mathrm{s})$ \\
\hline 0402 & $(0.91,0.28,-0.29)$ & 3.02 & -693 & -215 & 219 \\
0502 & $(0.94,0.19,-0.28)$ & 3.31 & -747 & -151 & 221 \\
0602 & $(0.96,0.09,-0.27)$ & 3.98 & -836 & -82 & 231 \\
0702 & $(0.97,-0.00,-0.25)$ & 3.98 & -844 & 2 & 217 \\
0803 & $(0.99,-0.02,0.15)$ & 3.98 & -861 & 22 & -132 \\
0903 & $(0.98,-0.12,0.17)$ & 3.63 & -813 & 101 & -140 \\
1004 & $(0.83,-0.15,0.53)$ & 2.90 & -618 & 110 & -397 \\
1104 & $(0.81,-0.24,0.54)$ & 2.40 & -546 & 164 & -363 \\
1204 & $(0.77,-0.34,0.54)$ & 2.05 & -484 & 210 & -335 \\
1304 & $(0.73,-0.43,0.53)$ & 1.86 & -437 & 253 & -315 \\
\hline
\end{tabular}

resolution of the instrument, and it cannot be reduced by tilting the $N$ axis toward the $L$ axis or $M$ axis. The alignment to a circle (not ellipse) as shown in the $L-M$ plot of Figure $8 \mathrm{~b}$ guarantees that the magnetic field orientation is approximately in either the $+\mathbf{N}$ or $-\mathbf{N}$ direction within this coordinate system.

It is worth noting that we need the energy information (i.e., the velocity vectors) to derive the correct result. If we use only the unit vectors instead of the velocity vectors, the minimum variance direction becomes $\pm(-0.99,0.11,-0.11)$, which is nearly $90^{\circ}$ off from the correct direction. Similarly, the intuitive method requires the energy information if the ring distribution is registered over different azimuthal sectors (as in the present case).

The minimum variance direction determined by the automatically filtered data (the same criterion as the previous event) is $\pm(-0.06,0.99,-0.09)$, which is again about $30^{\circ}$ off from the result obtained using the manual method. The observed ring distribution in the $L-M$ projection plots is not as well arranged to a circle in Figure $8 \mathrm{c}$ as in Figure 8b. Thus, the automatically filtered data again failed to determine the magnetic field direction to the same degree as the manual method.

Similar to the previous event, one can recognize high count rates during a short period corresponding to only one elevation scan (elevation $=7$ ) during the first 2 full scans in Figures 6 and 7 (1231:20 UT and 1234:30 UT, indicated by vertical arrows). Figure 9 shows the corresponding energy-mass matrix. Unlike the previous example, these short-time high count rates are repeated in the same direction (azimuth and elevation) at two consecutive full scans, which are more than 3 min apart. Therefore, they are most likely a spatial structure (i.e., keV ions flowing in a narrow direction (beam)) rather than a temporal structure (i.e., a narrow region of intense $\mathrm{keV}$ ion flux (boundary)), although the energies of these two beam-like ions are slightly different. The beam-like count is most intense at azimuth $=0$ /elevation $=7$ (direction is $(0.51,-0.12,-0.85)$ in MSO coordinates $)$, and it is registered in 

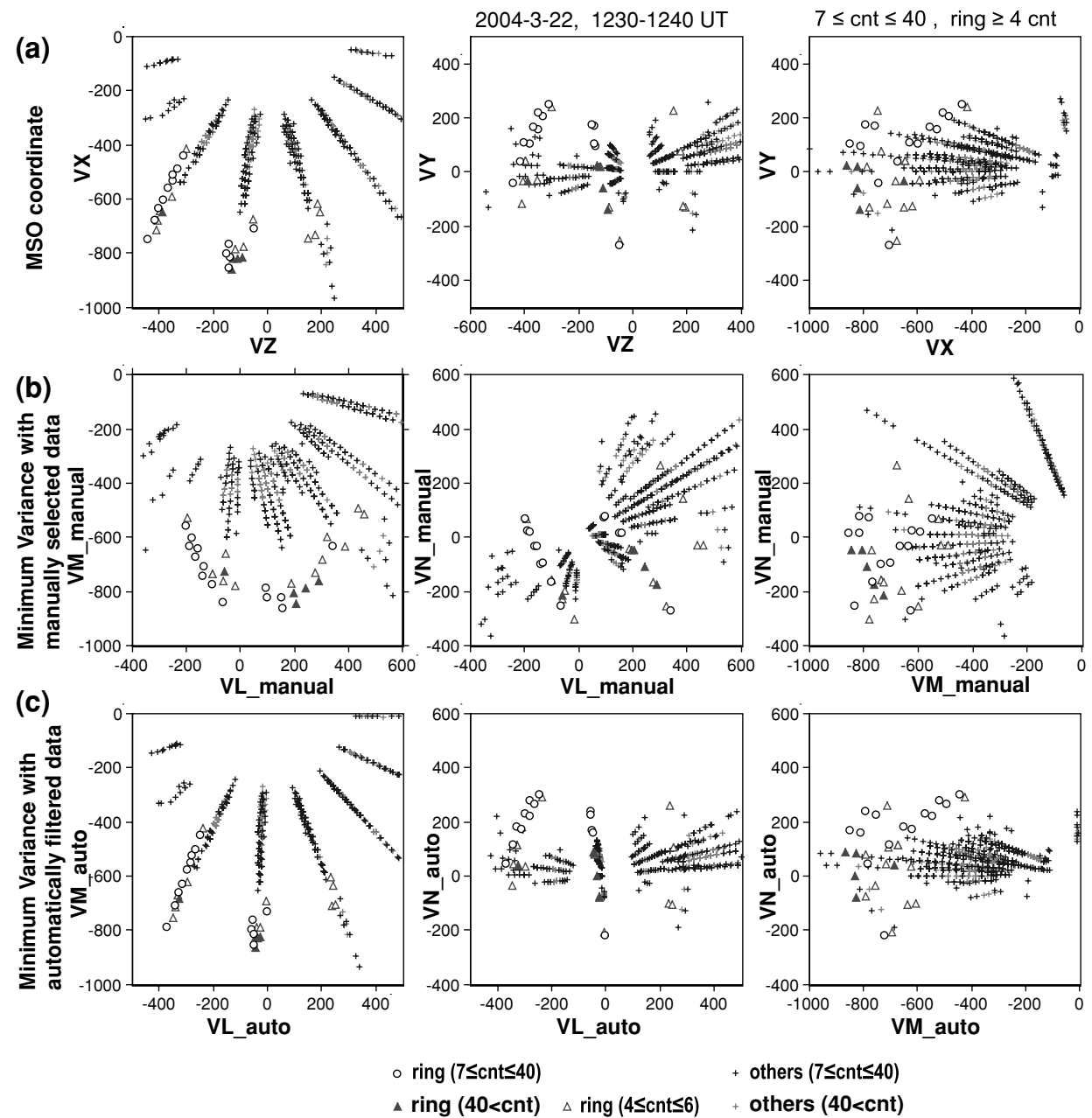

Figure 8. Velocity scatter plots of the ion data (in km/s) during 1230-1240 UT, 22 March 2004. The format is the same as Figure 5.

both the proton channel and the alpha particle channel. From its composition, these beam-like ions are of solar wind origin with energies of $2.5 \mathrm{keV}$ for protons and $4.5 \mathrm{keV} / \mathrm{q}(9 \mathrm{keV})$ for alpha particles, i.e., about 2.5 times the solar wind energy. The energy ratio indicates that both protons and alpha particles are accelerated to nearly same velocity $(650-700 \mathrm{~km} / \mathrm{s})$.

Since count rate registered at azimuth $=0$ is a summation of actual count rate reaching azimuth $=0$ and the contamination of all the other azimuths (from 1 to $15)$, it is generally not easy to extract the actual count only from azimuth $=0$. However, for this particular event at azimuth $=0$ /elevation $=7$, the count rate is largest among all azimuth (from 0 to 15), and hence this count rate cannot be the contaminated one from the other azimuth. The only other azimuth detecting 


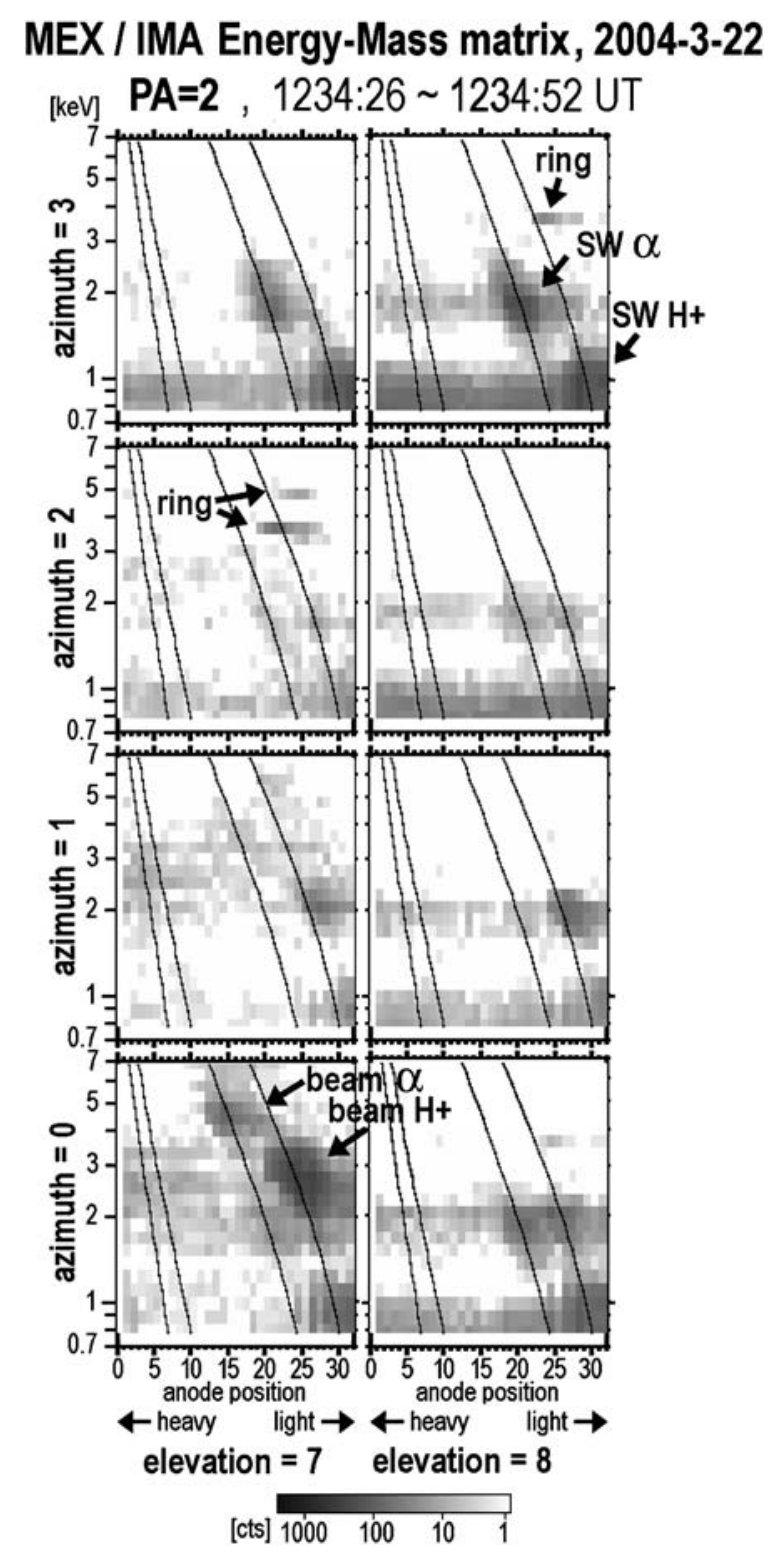

Figure 9. Energy-Mass matrices for elevation $=7$ (left: 1234:27-134:39 UT) and elevation $=8$ (right: 1234:39-134:51 UT) during the middle of Figure 6. The format of each panel is the same as Figure 3. From top to bottom are the azimuthal sectors 3, 2, 1, and 0. Azimuth $=3$ /elevation $=8$ corresponds to the solar wind direction. We show azimuth $=0$ because the beam-like ions are registered much stronger in azimuth $=0$ than in azimuth $=1$. The beam-like ions are not registered at other azimuth directions (cf. Figure 6). Therefore, the intense count at azimuth $=0$ is not due to contamination from the other sectors, but is a real feature. 
this beam-like ions is azimuth $=1$, but it is less intense than that registered at azimuth $=0$. Therefore, the count rate at azimuth $=1$ is most likely due to the effect of finite spread of direction. The center of the beam-like ions might even be shifted to azimuth $=15$ because, for this event, the quadrant of azimuth $=9-15$ and elevation $=0-7$ is blocked. However, the similar (but less intense) count at elevation $=8$ is registered only at azimuth $=0-1$ and not found at azimuth $=$ 13-14 (azimuth $=15$ is blocked at elevation $=8$ ). Therefore, the center of the beam-like count is more likely at azimuth $=0$. No matter where is the center direction, the registered directions of this beam-like ions are outside of the ring's plane.

The likely direction and energy of this beam-like ions (at azimuth $=0$ /elevation =7) correspond to a velocity of $(-360 \mathrm{~km} / \mathrm{s}, 80 \mathrm{~km} / \mathrm{s}, 600 \mathrm{~km} / \mathrm{s})$ in MSO coordinates, i.e., flowing nearly northward with a $30^{\circ}$ tilt toward the solar wind direction, and about $60^{\circ}$ in pitch angle. With such a large pitch angle, we should be able to detect a substantial number of counts from other directions at different energies which correspond to the gyration in velocity space. In the solar wind frame, the above velocity $(-360 \mathrm{~km} / \mathrm{s}, 80 \mathrm{~km} / \mathrm{s}, 600 \mathrm{~km} / \mathrm{s})$ corresponds to about $600 \mathrm{~km} / \mathrm{s}$ northward with the other velocity component about $100 \mathrm{~km} / \mathrm{s}$. Since the IMF lies within the $Y-Z$ plane about $30^{\circ}$ from the $Y$ axis, the expected velocity modulation is about $\pm 500 \mathrm{~km} / \mathrm{s}$ in the $Z$ direction $(0.5-4 \mathrm{keV}$ for a proton in the Martian rest frame). However, we did not identify the expected population in the expected directions.

The observed ring distribution becomes clearer as the spacecraft travels farther away from the bow shock. Only the highest energy part of the ring distribution is detected (i.e., the lower energy part disappeared in the both directions) during the first 2 scans after IMA entered the solar wind from the magnetosheath. Since the count of this highest energy part is near constant with more than 100 counts for all three scans, sensitivity of the instrument is high enough to observe the lower energy part in the first 2 scans. We cannot simply attribute this to the termination of the ion cycloid motion at the bow shock because the ring ions are flowing anti-sunward at all gyration phases. No answer to this issue has been forthcoming.

\subsection{OTHER EVENTS}

Ring distributions are often observed. Among 38 bow-shock crossings with PA = 2 mode during the first half of 2005, 30 cases show partial ring distributions from which the orientation of the ring plane can be estimated, and among these 30 cases, 7 cases show ring distributions that are extended in elevation as many sectors as the present case. For these 7 cases, the IMF direction can be derived with similar accuracy as in the examples presented here (see Section 4.2 below). For the rest of the 23 cases, the uncertainty in deriving the IMF direction might be larger, and this topic needs further investigation. 


\section{Discussion}

\subsection{Ring ShaPe}

The magnetic field direction can be estimated not only from the minimum variance direction but also from the direction that makes the ring-like distribution lie on a circle (not an ellipse) in the $L-M$ projection. If the $\mathbf{N}$ direction has an angle $d N$ from the magnetic field direction, the circular distribution in the $L-M$ projection becomes elliptic with a diameter ratio of $\cos (d N)$. Since we can observe only part of the distribution due to the low-energy instrumental limit, this method is less quantifiable than using the minimum variance direction. Yet the combination of these two methods improves the accuracy of determination of the orientation of the ring plane. Both Figures $5 \mathrm{~b}$ and $8 \mathrm{~b}$ show that the velocity scatter plot in the $L-M$ projection fits well into a (non-elliptic) circle. This guarantees that the minimum variance direction $\mathbf{N}$ is oriented approximately along the magnetic field.

Once the magnetic field direction is estimated, one can estimate the radius of the ring distribution compared to the solar wind velocity, and the offset of the fitted circle from zero velocity in the Martian rest frame in the $L-M$ plot. Radius and offset provide information on the initial velocity $\mathbf{V}_{0}$, and hence, the source population. Let us examine the 27 April 2005 event, in which the data lie well on the $L-M$ plane ( $V_{N}=0$ plane) as shown in Figure $5 \mathrm{~b}$. In this case, we just have to apply a least square fitting technique assuming a circle. Applying a linear weighting proportional to the count rate for each point, we obtain a center position $\left(V_{L}, V_{M}\right)$ $=(40 \mathrm{~km} / \mathrm{s},-430 \mathrm{~km} / \mathrm{s})$ and a radius of $320 \mathrm{~km} / \mathrm{s}$. This best-fit circle does not cross the origin (offset by about $110 \mathrm{~km} / \mathrm{s}$ ). However, an alternate fit circle with a radius of $420 \mathrm{~km} / \mathrm{s}$ (about solar wind velocity) does pass through the origin. Furthermore, the uncertainty in the normal direction $d N$ allows us to draw an ellipse (instead of a circle) passing through the origin. Thus, the least square fitting to a circle is not necessarily the appropriate solution.

The uncertainty in fitting to a circle/ellipse is more obvious for the 22 March 2004 event because $V_{N}$ is no longer zero for many points. Furthermore, the best fit in the $L-M$ projection could be an ellipse rather than a circle, with a shorter diameter in the $L$ direction than in the $M$ direction. In this case, the $N$ direction could be tilted toward the $\pm L$ direction. With such an uncertainty, we cannot derive $\mathbf{V}_{0}$ accurately enough to distinguish whether the source ions are newly ionized neutrals (zero velocity in Martian rest frame) or reflected solar wind.

No matter how we fit the data into a circle or an ellipse in the $L-M$ projection, the nearly entire circle/ellipse is located within $V_{M}<0$. This means that the ring population is always flowing in the $+M$ direction, which is mostly anti-sunward. This is also seen in the MSO coordinate velocity plot of Figure 5a: the fitted ellipse in the $X-Z$ plane stays entirely within $V_{X}<0$; i.e., the ions that form the ring distribution flow anti-sunward all of the time. 


\subsection{UNCERTAINTY}

The uncertainty of the derived IMF direction is determined by a combination of limitations in the spatial resolution of instrument and the data statistics. Contrary to high-angular resolution magnetic field data, the instrumental limit is very essential here because the azimuthal resolution of the IMA design is only $\pi / 8\left(22.5^{\circ}\right)$. Such a low angular resolution is a common limitation for almost all space plasma instruments. In the present case, small spread of the gyrating ions in the flowing directions slightly improves the resolution. If the direction of the gyrating ions is near the edge of one azimuthal sector, the ions are normally registered in the neighboring azimuthal sector as well. Therefore, the effective instrumental resolution is probably $15^{\circ}-20^{\circ}$. This is the basic accuracy in deriving the ring's normal direction, and the final accuracy (normally worse than this accuracy as shown below) is determined by the angular coverage of the detected ring ions in velocity space.

With IMA, one can only detect a partial ring (never complete) because of the low-energy limit of IMA (cf. Section 3.1). In such a case, separation between the minimum variance direction $(\mathbf{N})$ and the other variance directions ( $\mathbf{M}$ and $\mathbf{L}$ ) becomes an important factor in determining the uncertainty. For example, in both the $M-N$ plot and $L-N$ plot of Figure 8b, the data do not form a horizontal line, and their vertical scatter can stay within the same magnitude if we tilt the $M-N$ axis or $L-N$ axis. This is the degree of the uncertainty.

Three different rough estimates of the uncertainty for high-angular resolution data (like the magnetic field) are given in Sonnerup and Scheible (1998) by their equations (8.23)-(8.30) using the eigenvalues of the matrix that is used for the minimum variance analyses (cf. Table II). However, these formulas give zero uncertainty as long as the ring is observed in only one azimuthal sector, like the 27 April 2005 event (Figure 5b), because the minimum eigenvalue becomes identically zero for any 2-D limited data (i.e., limited in one azimuthal sector). Furthermore, for the 22 March 2004 event (Figures 8b and 8c), the automatic method yields a much smaller estimated error than the manual method, according to these formulas. This is because the error analyses assume that the majority of the data is relevant to the phenomena, while the automatic method cannot provide such clean data.

We are not aware of any formula that determines the uncertainty of minimum variance direction from low (and non-uniform) angular resolution data like in the present case. Therefore, we cannot tell how much the instrumental uncertainty (about 15-20 degrees) is enlarged or reduced through the analysis. Yet the instrumental uncertainty is most likely the limiting factor for at least the 27 April 2005 event (Figure 5b), because Figure $5 \mathrm{a}$ is only $20^{\circ}$ off from the anticipated direction (Figure 5b) and the fitting to a circle is apparently worse. The uncertainty for the 22 March 2004 event (Figure 8b) is not clear because the scatter of data in the $\mathbf{N}$ direction is not small as shown by both the $M-N$ plot and $L-N$ plot. Such a large scatter in the $\mathbf{N}$ direction is mainly caused by the $\pi / 8$ angular resolution in the sampling direction. 
One pedagogic method to evaluate the uncertainty is to modify the input data and see the result. Here we added one possible point (elevation $=2 /$ azimuth $=2$ at 1371 $\mathrm{eV}$ at $1334 \mathrm{UT}$ ) to the data in Table I and calculated the minimum variance direction. This direction is in the next azimuthal sector to the monochromatic sector (azimuth $=3$ ) found in Table I. The result is given in the third row of Table II (marked as "Table $I^{* * *}$ "). The minimum variance direction from this extended data is $12^{\circ}$ away from the minimum variance direction using only Table I data.

A similar method can be used to evaluate the uncertainty in the automatic method. If we include the data from 1334:00-1337:00 UT (this part contains many non-ring signatures) into the 1337:00-1357:00 UT data that was used in Section 3.1 , the resultant minimum variance direction (fourth row in Table II marked as " $7 \leq$ count $\leq 40^{* * * * *)}$ is about $60^{\circ}$ away from the direction obtained without adding the data. Thus, the automatic method is unstable.

Another pedagogic method for the uncertainty evaluation is to move the $N$ direction and see the change in circle fitting. For example, the $L-M$ plots of Figure $8 \mathrm{~b}$ shows a rather good circle fit, but it is also possible to fit the data to an ellipse with the diameter ratio $\cos \left(30^{\circ}\right)=0.87$. In other words, tilting the data $30^{\circ}$ in the $L-N$ direction still fits a circle (not a ellipse) that passes through the origin. Such a tilt does not affect the spread of $V_{N}$ data in the $L-N$ plot. In other words, the $\pi / 8$ angular resolution of the instrument can easily be worsened to $30^{\circ}$ through the spread of $V_{N}$ if the ring is registered in more than one azimuthal sector.

\subsection{Source Population}

That the ring distribution is restricted to a plane indicates that the source population is either beam-like (just for the initial velocity) or has zero velocity in the Martian rest frame. The ring distribution is often observed many minutes after the bow shock crossing, with the flow direction mainly in the anti-sunward (e.g., see $V_{X}$ values in Tables I and III). For the case of the 27 April 2005 event, a ring distribution with nearly zero parallel velocity was observed more than $1000 \mathrm{~km}$ upstream of where IMA crossed the bow shock (upper right panel of Figure 1). Since both the solar wind and IMF were stable during the observation period (cf. lower panel of Figure 1, and the last paragraph in Section 2.1), it is impossible that the bow shock moved outward significantly during this event. With the observed solar wind velocity $(>400 \mathrm{~km} / \mathrm{s})$, the bow shock is expected to be closer to Mars than the model location shown in Figure 1. Therefore, the ring distribution existed more than $1000 \mathrm{~km}$ upstream the bow shock. In this sense, the formation mechanism of the ring distribution upstream of Mars is quite different from the Earth's case where the ring distribution is observed only inside the foot region (e.g., Sckopke et al., 1990).

As mentioned in Section 4.1, all ions that belong to the ring distribution flow anti-sunward even for those that are not detected by IMA. Furthermore, a positive 
$V_{Y}$ in Table I (bow shock position is $Y<0$ ) means that the ions are coming from position with a larger $|Y|$ than the MEX location. The estimated IMF direction is such that the spacecraft is not magnetically connected to any part of the bow shock during the last scan at 1354-1357 UT. From these facts, the ring distribution at 1354-1357 UT, with nearly zero parallel velocity, cannot be traced back to the bow shock even considering a finite gyroradius of the ring protons. In other words, the source population must have been transported to the upstream region from the bow shock beyond the proton gyroradius, and such transport is possible only if the source population is not ionized, i.e., in the form of the neutral hydrogen atoms. The atoms escape outward from the bow shock and start the cycloid motion after they are ionized, like the comet case. This is the so called ion pick-up by the solar wind.

According to our present knowledge, there are two types of sources for massive amounts of neutral hydrogen atoms escaping beyond the bow shock into the upstream region of the solar wind. The most probable source is the hydrogen corona that hydrostatically extends from the exobase (e.g., Lammer et al., 2005). Since the distance between the Martian exobase and its bow shock is closer than that between the Earth's exobase and its bow shock, it is possible that the Martian hydrogen corona extends beyond the bow shock. The part of the hydrogen corona that is exposed to the solar wind is ultimately picked-up by the solar wind.

No solid observation exists on how far the hydrogen corona extends. If the source population of the ring distribution is the hydrogen corona, the distribution of the pick-up ions can provide the extent of the hydrogen corona (Barabash et al., 1991; Barabash and Lundin, 1993; Dubinin et al., 1994, 1995). This information determines the efficiency of the entire pick-up loss of the hydrogen from Mars. Since the pick-up loss is believed to contribute a large part to the hydrogen loss from Mars, deriving the coronal extent is important in understanding the evolution of the Martian atmosphere (and hence, Mars itself).

The other possible source is the newly found energetic neutral atom (ENA) jet emitting only from the subsolar bow shock region into a narrow angle mainly in the $Y$ and $Z$ direction (Futaana et al., 2006; Gunell et al., 2006). This ENA source is believed to be of solar wind origin from the energy information. The question is if the flux of the ENA jet and its ionization rate is high enough to produce the necessary newly born ions for the detected ring distribution. Unfortunately, the ENA instruments (NPD and NPI) on board MEX were turned off during both events, and we have to employ the preliminary statistics with the ENA flux of (4-7) $\times 10^{5} \mathrm{~cm}^{-2} \mathrm{str}^{-1} \mathrm{~s}^{-1}$ (Futaana et al., 2006). This corresponds to about two orders of magnitude less than the solar wind flux if we assume the angular extent of the ENA beam as about 1 str, and cannot be at moment dismissed because the flux of the ring distribution is also two orders of magnitude less than the solar wind flux (last paragraph in Section 3.1). Further examinations of this scenario require detailed modeling, which is beyond the scope of this paper. If we can eliminate this possibility, the frequent observation of the ring distribution provides a new way to study the Martian exosphere. 
One may wonder whether the short-duration high count populations of at around 1335:10 UT (azimuth $=3$ /elevation $=4$ ) in Figure 2 and at around 1231:20 UT and 1234:30 UT (azimuth $=0 /$ elevation $=7$ ) in Figure 6 are related to the ring distribution. The beam-like population in Figure 6 is directed in a direction away from the fitted circle or ellipse of the ring in velocity space, and it is difficult to consider a direct relation between this beam-like population and the ring population. On the other hand, the single high count event in Figure 2 falls into the fitted circle of the ring in velocity space, with the exception that this high count population has a non-zero parallel velocity (non-zero $V_{N}$ ), which may be attributed to instrumental uncertainty. Since this high count population have the same $V_{X}$ component as the solar wind velocity pointing the anti-sunward direction with a minor (positive) $V_{Z}$ component, this population cannot be the source of the ring distribution, but it could be a result of the ring distribution.

\section{Conclusions}

Using two examples (27 April 2005 event and 22 March 2004 event) of MEX/IMA observations, we have shown that the approximate IMF orientation can be derived from the 3-D ion velocity distribution as measured by the IMA instrument when the instrument observed ring-like distributed protons in a plane in velocity space. Since the ring distribution represents a gyration trajectory (cycloid motion) of an ensemble of ions with nearly the same initial velocity (beam-like or zero velocity), the ring's plane must be perpendicular to the IMF. Such ring distributions are observed (in the spectrogram like Figures 2 and 7) in 31 cases among 39 bow-shock crossings with the operation mode that can detect the solar wind protons during 2004 and first half of 2005.

On 27 April 2005 (1337-1357 UT) IMA detected the ring distribution in an ideal orientation, i.e., only in azimuthal sector 3 and for elevation scans from 2 through 15 (Table I). On 22 March 2004 (1230-1340 UT) the ring distribution was detected with a more general orientation, i.e., azimuthal sector 2 for elevation sector 2 to 7 , azimuthal sector 3 for elevation sector 8 and 9, and azimuthal sector 4 from elevation sector 10 to 13 (Table III).

Three different methods are demonstrated to derive the IMF orientations for both events (Figures 5 and 8 ). The intuitive method and the minimum variance method using manually selected data agrees with each other, constructing a local Cartesian (LMN) coordinate that arranges the ring-like distribution along a circle in the $L-M$ projection with a constant $V_{N}$. The result guarantees that the magnetic field is pointing either in the $+\mathbf{N}$ or $-\mathbf{N}$ direction with the angular accuracy mainly determined by the instrumental limit and the statistics.

The minimum variance direction obtained from the automatically filtered data is more than $30^{\circ}$ away from the direction derived from the manually selected data even for an ideal case presented here. The ring distribution projected into the derived 
LMN coordinates is not as well arranged by the automatic method as by the manual method. This is because the simple automatic filters cannot provide an appropriately cleaned dataset that consist only of the ring distribution. Since the ring distribution is just one of the secondary populations seen in the solar wind, the solar wind and other components (e.g., beam-like population) severely deform the minimum variance direction.

The derived IMF direction (unit vector with arbitrary sign) in MSO coordinates is $\pm(0.34,0.94,-0.003)$ for the 27 April 2005 event $\left(20^{\circ}\right.$ from the $Y$ axis within the $X-Y$ plane $)$, and $\pm(-0.01,0.88,0.47)$ for the 22 March 2004 event $\left(30^{\circ}\right.$ from the $Y$ axis within the $Y-Z$ plane). The uncertainty in the derived IMF is determined by the instrumental angular resolution $\left(15-20^{\circ}\right)$ for the 27 April 2005 event, but is worsened to about $30^{\circ}$ for the 22 March 2004 event.

The source of the ring distribution is most likely newly ionized hydrogen atoms (and picked-up by the solar wind) because the ring distribution is detected beyond the finite gyro-radius distance from the bow shock. The hydrogen corona is a strong candidate while we cannot eliminate at moment the newly found ENA jet as another possible candidate. The high count protons observed near the bow shock (at around 1335:10 UT), which shares the same velocity space as the ring distribution, cannot be the ultimate source of the ring distribution, but they could be the result of the ring distribution.

\section{Acknowledgements}

The Mars Express mission is the first European mission to the red planet managed by European Space Agency (ESA). The ASPERA-3 experiment on the Mars Express mission is a joint effort between 15 laboratories in 10 countries, all sponsored by their national agencies. We thank all these agencies as well as the various departments/institutes hosting these efforts. Work at the main PI-institute for the ASPERA-3 experiment is supported by the Swedish National Space Board, while the work at the main PI for the ELS instrument is supported by National Aeronautics and Space Administration (NASA) contract NASW-00003 in the United States. Y. Futaana is supported by Postdoctoral Fellowships for Research Abroad of the Japan Society for the Promotion Science.

\section{References}

Acuña, M. H., Connerney, J. E. P., Wasilewski, P., Lin, R. P., Anderson, K. A., Carlson, C. W., et al.: 1998, Science 279(5357), 1676.

Barabash, S., and Lundin, R.: 1993, Geophys. Res. Lett. 20, 787.

Barabash, S., Dubinin, E., Pissarenko, N., Lundin, R., and Russell, C. T.: 1991, Geophys. Res. Lett. 18,1805 . 
Barabash, S., Lundin, R., Andersson, H., Gimholt, J., Holmstrom, M., Norberg, O., et al: 2004, in Mars Express: The Scientific Payload, ESA SP-1240, pp. 121-139.

Dubinin, E., Lundin, R., Koskinen, H., and Norberg, O.: 1993, Geophys. Res. Lett. 98(A4), 5617.

Dubinin E., Obod, D., Pedersen, A., and Grard, R.: 1994, Geophys. Res. Lett. 21, 2769.

Dubinin E., Obod, D., Lundin, R., and Grard, R.: 1995, Adv. Space Res. 15, 8(9), 423.

Fedorov, A., Budnik, E., Sauvaud, J.-A., Mazelle, C., Barabash, S., Lundin, R., et al.: 2006, Icarus 182(2), 329, doi: 10.1016/j.icarus.2005.09.021.

Futaana, Y., Barabash, S., Grigoriev, A., Holmström, M., Kallio, E., C:son Brandt, P., et al.: 2006, Icarus 182(2), 413-423, doi: 10.1016/j.icarus.2005.08.024.

Futaana, Y., Machida, S., Saito, Y., Matsuoka, A., and Hayakawa, H.: 2003, J. Geophys. Res. 108(A10), doi: 10.1029/2002JA009366.

Gunell, H., Brinkfeldt, K., Holmström, M., Brandt, P., Barabash, S., Kallio, E., et al.: 2006, Icarus 182(2), 431-438, doi: 10.1016/j.icarus.2005.10.027.

Möbius, E., Kucharek, H., Mouikis, C., Geogescu, E., Kistler, L. M., et al.: 2001, Ann. Geophys. 19, 1411.

Mukai, T., Miyake, W., Terasawa, T., Kitayama, M., and Hirao, K.: 1986a, Nature 321, 299.

Mukai, T., Miyake, W., Terasawa, T., Kitayama, M., and Hirao, K.: 1986b, Geophys. Res. Lett. 13, 829.

Lammer, H., Lichtenegger, H. I. M., Penz, T., Amerstorfer, U. V., Kolb, C., and Biernat H. K.: 2005, in H. K. Biernat, H. Lammer, D. F. Vogl, and S. Mühlbachler (eds.), Research Signpost, Trivandrum, India, p. 209.

Lundin, R., Barabash, S., Andersson, H., Holmström, M., Grigoriev, A., Yamauchi, M., et al.: 2004, Science 305, 1933.

Paschmann, G., Sckopke, N., Papamastorakis, I., Asbridge, J. R., Bame, S. J., and Gosling, J. T.: 1981, J. Geophys. Res. 86, 4355.

Sckopke, N., Paschmann, G., Bame, S. J., Gosling, J. T., and Russell, C. T.: 1983, J. Geophys. Res. $\mathbf{8 8}, 6121$.

Sckopke, N., Paschmann, G., Brinca, A. L., Carlson, C. W., and Luhr, H.: 1990, J. Geophys. Res. 95, 6337.

Sonnerup, B. U. O., and Cahill, Jr., L. J.: 1967, J. Geophys. Res. 72, 171.

Sonnerup, B. U. O., and Scheible, M.: 1998, in G. Paschmann, and P. W. Daly (eds.), ISSI Scientific Report, ESA Publications Division, Noordwijk, The Netherlands.

Terasawa, T., Mukai, T., Miyake, W., Kitayama, M., and Hirao, K.: 1986, Geophys. Res. Lett. $13,837$.

Winningham, J. D., Frahm, R. A., Sharber, J. R., Coates, A. J., Linder, D. R., Soobiah, et al.: 2006, Icarus 182(2), 360, doi: 10.1016/j.icarus.2005.10.033. 